\title{
Importance of eliminating potential dental focal infection before the first cycle of chemotherapy in patients with hematologic malignancy
}

\author{
Yasuyuki Shimada $^{1,2}$ (D) Yumiko Nakagawa ${ }^{3} \cdot$ Kazuki Ide $^{2,4,5} \cdot$ Izumi Sato $^{4,6}$. \\ Shotaro Hagiwara $^{7}$ • Hiroshi Yamada ${ }^{2}$. Yohei Kawasaki ${ }^{2,4}$ - Yutaka Maruoka ${ }^{1,8}$
}

Received: 11 April 2016 / Accepted: 17 February 2017 /Published online: 27 February 2017

(C) The Author(s) 2017. This article is published with open access at Springerlink.com

Letter to the Editor,

The oral cavity is a potential infection site in patients undergoing immunosuppressive therapy [1]. These patients are more susceptible to systemic infection, particularly those who need chemotherapy for hematologic malignancies [2]. Many dentists find it difficult to select an approach for dental focal infection in these patients [1,2]. Although there is some literature assessing oral health in patients undergoing chemotherapy or pre-hematopoietic stem cell transplantation [3], these patients had already experienced adverse effects of chemotherapy, making it difficult to interpret the effect of dental treatment. Tooth extraction is a common surgical procedure

Yasuyuki Shimada

yasuyuki.shimada.ys@gmail.com

1 Department of Oral and Maxillofacial Surgery, Center Hospital of the National Center for Global Health and Medicine, 1-21-1 Toyama, Shinjuku-ku, Tokyo 162-8655, Japan

2 Department of Drug Evaluation and Informatics, School of Pharmaceutical Sciences, University of Shizuoka, Shizuoka, Japan

3 AIDS Clinical Center, National Center for Global Health and Medicine, Tokyo, Japan

4 Department of Pharmacoepidemiology, Graduate School of Medicine and Public Health, Kyoto University, Kyoto, Japan

5 Center for the Promotion of Interdisciplinary Education and Research (C-PIER), Kyoto University, Kyoto, Japan

6 Keihanshin Consortium for Fostering the Next Generation of Global Leaders in Research (K-CONNEX), Kyoto, Japan

7 Division of Hematology, Department of Internal Medicine, Center Hospital of the National Medical Center for Global Health and Medicine, Tokyo, Japan

8 Oral and Maxillofacial Surgery, Department of Oral Restitution, Division of Oral Health Sciences, Graduate School, Tokyo Medical and Dental University, Tokyo, Japan performed to eliminate dental focal infection, and it carries a risk of perioperative surgical site infection (SSI) [1]. Although the frequency of tooth extraction-associated SSI is $<5 \%$ in healthy individuals [4], it may be increased in patients with hematologic malignancy.

We retrospectively reviewed the records of 160 patients with hematologic malignancy who visited the Department of Oral and Maxillofacial Surgery, Center Hospital, National Center for Global Health and Medicine, Tokyo, Japan, over a 2-year period to clarify the incidence of oral adverse events if dental problems are treated before the first chemotherapy cycle. Seventy-five patients were treated before the first cycle. Dental treatments were performed, and oral health status was assessed after chemotherapy. The observation period was the number of days from the start of the first chemotherapy cycle to just before the start of the second cycle. In cases where the cycle was unclear (e.g., when a single agent was administered repeatedly), the observation period was 30 days from chemotherapy initiation (i.e., the period deemed by hospital management to constitute adequate follow-up).

Nine $(12.0 \%)$ patients experienced oral adverse events after chemotherapy. No significant difference was found between the groups with and without tooth extraction $(P=0.28)$. The most frequently observed symptom was periodontitis aggravation $(n=3,33.0 \%)$. Although all three patients were deemed to require extraction at the first stomatognathic screening, a decision not to proceed was made in two cases owing to a tight chemotherapy schedule. However, inflammation steadily worsened thereafter. Remarkably, only two of 28 $(7.1 \%)$ tooth extraction cases had a poor outcome, regardless of the operative stress (surgical or simple extraction). Oral antibiotics (penicillin or cephem) were administered starting just after surgery in all extraction cases and continued at least for 3 days. If oral administration was impossible, the intravenous route was used. The median (min-max) white blood cell 
and absolute neutrophil counts at extraction were $5685 / \mu \mathrm{L}$ $\left(720-123,300 \times 10^{3} / \mu \mathrm{L}\right)$ and $3889 / \mu \mathrm{L}(36-12,330 / \mu \mathrm{L})$, respectively. Sixty-six $(88.0 \%)$ patients did not experience oral adverse events and were able to continue chemotherapy without delay.

Oral mucositis is a well-known adverse event in patients undergoing chemotherapy [5], and was observed frequently in our patients. However, the frequency was lower compared with a previous report [5]. Although further investigation is necessary to clarify the effectiveness, intraoral icing during chemotherapy was performed for all patients, and this may protect against stomatitis.

Stomatognathic region surgery is normally associated with postoperative complications such as pain, swelling, and trismus. These are detrimental to oral hygiene and change the oral bacterial flora, which may aggravate periodontitis and stomatitis [6,7]. Although these symptoms are not directly related to the surgical procedure, we would classify them as adverse events because they almost certainly affect quality of life. Periodontitis or stomatitis may lead to a delay or interruption of chemotherapy due to cellulitis, as well as cause life-threatening sepsis in immunosuppressed patients [2]. Therefore, we recommend that potential sources of infection in the oral cavity be identified and eliminated before the first chemotherapy cycle when possible. In addition, we recommend regularly investigating oral health status after chemotherapy initiation and performing periodontal treatment and professional oral health care as necessary.

Only one $(3.6 \%)$ of the 28 patients who underwent tooth extraction before chemotherapy developed an SSI. This frequency is surprisingly low. The median (min-max) time allowed between tooth extraction and chemotherapy initiation was $13(0-66)$ days. Although we divided this period into six 10-day groups, we did not find a statistically significant relationship with oral adverse events $(P=0.37)$. As mentioned above, the risk of SSI associated with tooth extraction in a healthy individual is $<5 \%$ [4], which is similar to our findings. Table 1 compares the relative frequencies of adverse events in our patients with those reported in the literature [1, 2, 8-10]. Consistent with our findings, the proportion of patients with SSI associated with tooth extraction is low $(<5 \%)$ and no systemic infection associated with tooth extraction was described in most reports. In addition, none of the patients who received treatment other than tooth extraction at our institution experienced an adverse event during the observation period. These observations suggest that the presence of hematologic malignancy does not adversely affect recovery from dental treatment including tooth extraction. However, Akashi et al. reported a high SSI frequency associated with tooth extraction (10\%) that evolved into sepsis, and Raut et al. and Haytac et al. reported an inability to initiate chemotherapy as scheduled due to SSI associated with tooth extraction in 5.8 and $4.2 \%$ of cases, respectively. Unlike our cohort, the abovementioned patients underwent dental surgery during hematopoietic stem cell transplantation and had already experienced adverse effects due to pancytopenia caused by potent chemotherapy. Although further investigation is necessary to clarify the risk factor(s) for oral adverse events, chemotherapy intensity or type may affect the dental prognosis. The small number of patients who underwent tooth extraction and had a poor outcome may also have influenced the accuracy of our statistical analysis. We may be able to include more in our next report to clarify the risks associated with tooth extraction in this population.

In summary, we did not find a significant relationship between tooth extraction before the first chemotherapy

Table 1 Relative frequencies of oral adverse events compared with other countries in patients with hematologic malignancy

\begin{tabular}{|c|c|c|c|c|c|c|}
\hline & Overholser CD & Williford SK & Raut A & Haytac MC & Akashi M & Shimada Y \\
\hline Year & 1982 & 1989 & 2001 & 2004 & 2013 & 2016 \\
\hline Nationality & USA & USA & USA & TUR & JPN & JPN \\
\hline Total no. of pt. & 28 & 26 & 388 & 124 & 37 & 160 \\
\hline No. of before chemotherapy pt. & - & 5 & 2 & 0 & 0 & 75 \\
\hline Age (median) & - & - & - & 7 & 50 & 63 \\
\hline $\operatorname{Sex}(m: f)$ & $1: 1.3$ & - & - & $1.2: 1$ & $1.6: 1$ & $1.8: 1$ \\
\hline No. of tooth extraction pt. & $28 / 28$ & $26 / 26$ & $69 / 388$ & $48 / 124$ & $10 / 37$ & $28 / 75$ \\
\hline Total no. of pt. with oral adverse event after chemotherapy & $1 / 28(3.6 \%)$ & $7 / 26(27 \%)$ & $9 / 69(13 \%)$ & $10 / 48(21 \%)$ & $2 / 37(5.4 \%)$ & $9 / 75(12 \%)$ \\
\hline Total no. of pt. with systemic infection of oral origin & $0 / 28(0 \%)$ & $0 / 26(0 \%)$ & $0 / 69(0 \%)$ & $0 / 48(0 \%)$ & $2 / 37(5.4 \%)$ & $0 / 75(0 \%)$ \\
\hline Delay of chemotherapy & $0 / 28(0 \%)$ & $0 / 26(0 \%)$ & $4 / 69(5.8 \%)$ & $2 / 48(4.2 \%)$ & $0 / 37(0 \%)$ & $0 / 75(0 \%)$ \\
\hline SSI associated with tooth extraction & $1 / 28(3.6 \%)$ & $1 / 26(3.8 \%)$ & $0 / 69(0 \%)$ & $0 / 48(0 \%)$ & $1 / 10(10 \%)$ & $1 / 28(3.6 \%)$ \\
\hline
\end{tabular}


cycle and oral adverse events thereafter. Dental focal infection may adversely affect patients with hematologic malignancy, so dental infections should ideally be identified and eliminated before initiating chemotherapy. Specialists should pay close attention to oral health in patients with hematologic malignancy so their treatment can be appropriately sequenced.

Acknowledgments This work was supported in part by Grants-in-Aid for Research from the National Center for Global Health and Medicine (28-1105). We would like to thank Editage (www.editage.jp) for English language editing.

Author contributions YS, YN, KI, IS, SH, HY, YK, and YM conceived and designed the study; YS, YN, KI, and YK analyzed the data; YS and KI wrote the paper; all authors read and approved the final version submitted.

\section{Compliance with ethical standards}

Conflict of interest The authors declare that they have no conflict of interest.

Ethical approval All investigations were performed according to protocols that were reviewed and approved by the ethical committee of NCGM (NCGM-G-00179-00). We also conducted the study in accordance with the Helsinki declaration. For this type of study, formal consent is not required.

Open Access This article is distributed under the terms of the Creative Commons Attribution-NonCommercial 4.0 International License (http://creativecommons.org/licenses/by-nc/4.0/), which permits any noncommercial use, distribution, and reproduction in any medium, provided you give appropriate credit to the original author(s) and the source, provide a link to the Creative Commons license, and indicate if changes were made.

\section{References}

1. Raut A, Huryn JM, Hwang FR, Zlotolow IM (2001) Sequelae and complications related to dental extractions in patients with hematologic malignancies and the impact on medical outcome. Oral Surg Oral Med Oral Pathol Oral Radio Endod 92:49-55. doi:10.1067/ moe.2001.113588

2. Akashi M, Shibuya Y, Kusumoto J et al (2013) Myelosuppression grading of chemotherapies for hematologic malignancies to facilitate communication between medical and dental staff: lessons from two cases experienced odontogenic septicemia. BMC Oral Health 13:41. doi:10.1186/1472-6831-13-41

3. Tsuji K, Shibuya Y, Akashi M et al (2015) Prospective study of dental intervention for hematopoietic malignancy. J Dent Res 94: 289-296. doi:10.1177/0022034514561768

4. Chuang SK, Perrott DH, Susarla SM, Dodson TB (2008) Risk factors for inflammatory complications following third molar surgery in adults. J Oral Maxillofac Surg 66:2213-2218. doi:10.1016/ j.joms.2008.06.067

5. Niscola P, Romani C, Cupelli L et al (2007) Mucositis in patients with hematologic malignancies: an overview. Haematologica 92: 222-231. doi:10.3324/haematol.10232

6. Brickley M, Armstrong R, Shepherd J, Kay E (1995) The relevance of health state utilities to lower third molar surgery. Int Dent J 45: $124-128$

7. Kojima A, Ekuni D, Mizutani S et al (2013) Relationships between self-rated oral health, subjective symptoms, oral health behavior and clinical conditions in Japanese university students: a crosssectional survey at Okayama University. BMC Oral Health 13:62. doi:10.1186/1472-6831-13-62

8. Overholser CD, Peterson DE, Bergman SA, Williams LT (1982) Dental extractions in patients with acute nonlymphocytic leukemia. J Oral Maxillofac Surg 40:296-298. doi:10.1016/0278-2391(82) 90222-1

9. Williford SK, Salisbury PL 3rd, Peacock JE Jr, Cruz JM, Powell BL, Lyerly ES, Capizzi RL (1989) The safety of dental extractions in patients with hematologic malignancies. J Clin Oncol 7:798-802

10. Haytac MC, Dogan MC, Antmen B (2004) The results of a preventive dental program for pediatric patients with hematologic malignancies. Oral Health Prev Dent 2:59-65 